\title{
TV Corvi revisited: Precursor and superhump period derivative linked to the disk instability model
}

\author{
M. Uemura ${ }^{1,2}$, R. E. Mennickent ${ }^{1}$, R. Ishioka ${ }^{3}$, A. Imada ${ }^{3}$, T. Kato ${ }^{3}$, D. Nogami ${ }^{4}$, R. Stubbings ${ }^{5}$, S. Kiyota ${ }^{6}$, \\ P. Nelson ${ }^{7}$, K. Tanabe ${ }^{8}$, B. Heathcote ${ }^{9}$, and G. Bolt ${ }^{10}$ \\ 1 Departamento de Física, Facultad de Ciencias Físicas y Matemáticas, Universidad de Concepción, Casilla 160-C, \\ Concepción, Chile \\ e-mail: muemura@cepheid.cfm.udec.cl \\ 2 Yukawa Institute for Theoretical Physics, Kyoto University, Kyoto 606-8502, Japan \\ 3 Department of Astronomy, Kyoto University, Sakyou-ku, Kyoto 606-8502, Japan \\ 4 Hida Observatory, Kyoto University, Kamitakara, Gifu 506-1314, Japan \\ 519 Greenland Drive, Drouin 3818, Victoria, Australia \\ 6 Variable Star Observers League in Japan (VSOLJ), 1-401-810 Azuma, Tsukuba 305-0031, Japan \\ 7 RMB 2493, Ellinbank 3820, Australia \\ ${ }^{8}$ Department of Biosphere-Geosphere Systems, Faculty of Informatics, Okayama University of Science, Ridaicho 1-1, \\ Okayama 700-0005, Japan \\ 9 Barfold Observatory, Mia Mia, Victoria, Australia \\ 10295 Camberwarra Drive, Craigie 6025, Western Autralia
}

Received 14 September 2004 / Accepted 1 November 2004

\begin{abstract}
We report optical photometric observations of four superoutbursts of the short-period dwarf nova TV Crv. This object experiences two types of superoutbursts; one with a precursor and the other without. The superhump period and period excess of TV Crv are accurately determined to be $0.065028 \pm 0.000008 \mathrm{~d}$ and $0.0342 \pm 0.0021$, respectively. This large excess implies a relatively large mass ratio of the binary components $\left(M_{2} / M_{1}\right)$, though it has a short orbital period. The two types of superoutbursts can be explained by the thermal-tidal instability model for systems having large mass ratios. Our observations reveal that superhump period derivatives are variable in distinct superoutbursts. The variation is apparently related to the presence or absence of a precursor. We propose that the superhump period derivative depends on the maximum disk radius during outbursts. We investigate the relationship of the type of superoutbursts and the superhump period derivative for known sources. In the case of superoutbursts without a precursor, superhump period derivatives tend to be larger than those in precursor-main type superoutbursts, which is consistent with our scenario.
\end{abstract}

Key words. accretion, accretion disks - binaries: close - novae, cataclysmic variables - stars: dwarf novae stars: individual: TV Crv

\section{Introduction}

SU UMa-type stars form a sub-group of dwarf novae characterized by the appearance of long and bright "superoutbursts", during which periodic modulations, "superhumps", are observed (Warner 1985). Superhumps have periods slightly longer than orbital periods, which can be explained by a beat phenomenon of a precessing tidally-distorted eccentric disk. According to the tidal instability theory, an accretion disk becomes unstable against a tidal perturbation from a secondary star when the disk reaches the 3:1 resonance radius (Whitehurst 1988). In conjunction with the thermal instability model for (normal) dwarf nova outbursts, the model for superoutbursts is called the thermal-tidal instability (TTI) model (Osaki 1989).
Superoutbursts are sometimes associated with a precursor typically lasting one or two days. This precursor phenomenon is actually expected from the TTI model. The precursor is considered to be a normal outburst leading to an expansion of the accretion disk over the 3:1 resonance radius and triggering a superoutburst. Growing superhumps have been detected during a decay phase from the precursor in T Leo (Kato 1997), V436 Cen (Semeniuk 1980), and GO Com (Imada et al. 2004). These growing superhumps provide evidence for the TTI model since a system is predicted to reach a supermaximum with the growth of an eccentric disk. On the other hand, the original TTI model cannot explain gradually growing superhumps even after supermaxima without a precursor, which are also frequently observed (Smak 1996). 
Osaki \& Meyer (2003) propose a refinement of the original TTI model with the idea that the accretion disk can pass the $3: 1$ resonance radius and reach the tidal truncation radius. The dammed matter at the tidal truncation radius causes a gradual decay without a precursor. This refined TTI model predicts that SU UMa stars having a large mass ratio $\left(q=M_{2} / M_{1}\right.$, where $M_{1}$ and $M_{2}$ are the masses of a white dwarf and a secondary star, respectively) can show both types of superoutbursts, that is, those with and without a precursor. This idea should be examined by observations of the early evolution of superoutbursts and superhumps.

The superhump period $\left(P_{\mathrm{SH}}\right)$ in SU UMa stars generally decreases through a superoutburst with a period derivative of order $\dot{P}_{\mathrm{SH}} / P_{\mathrm{SH}} \sim-10^{-5}$ (Warner 1985; Patterson et al. 1993). A simple dynamical treatment for the tidal instability shows that the precession rate of the eccentricity wave is proportional to $r^{1.5}$, where $r$ is the disk radius (Osaki 1985). The shortening of $P_{\mathrm{SH}}$ can, hence, be understood with the shrink of the disk during a superoutburst. Hydrodynamical simulations also show that the precessing eccentricity wave propagates inward, which causes the period shortening of superhumps (Lubow 1992; Whitehurst 1994).

On the other hand, several short-period SU UMa stars showing positive $\dot{P}_{\mathrm{SH}} / P_{\mathrm{SH}}$ have been discovered since mid90's (Howell et al. 1996a; Kato et al. 2003c). WZ Sge-type stars, in particular, tend to show positive $\dot{P}_{\mathrm{SH}} / P_{\mathrm{SH}}$ (e.g. Howell et al. 1996a; Kato et al. 1997). The situation becomes more complicated because ultra-short period systems, V485 Cen and EI Psc also show positive $\dot{P}_{\mathrm{SH}} / P_{\mathrm{SH}}$ (Olech 1997; Uemura et al. 2002a). These two sources have quite large mass ratios ( $q \sim 0.2$ ), though WZ Sge stars have quite small mass ratio $(q \sim 0.01)$. Based on the discussions for ordinary negative $\dot{P}_{\mathrm{SH}} / P_{\mathrm{SH}}$, the positive $\dot{P}_{\mathrm{SH}} / P_{\mathrm{SH}}$ has been proposed to arise due to an expansion of the disk or an outward-propagation of the eccentricity wave (Baba et al. 2000; Kato et al. 2004a). It is, however, poorly understood why the outward propagation can occur only in the short-period systems regardless of their mass ratio (Ishioka et al. 2003).

TV Crv is known as an SU UMa-type dwarf nova having a short orbital period of $0.06288 \pm 0.00013 \mathrm{~d}$ (Woudt \& Warner 2003). The historical discovery of this object is summarized in Levy et al. (1990). Howell et al. (1996b) reported superhumps with a period of $0.0650 \pm 0.0008 \mathrm{~d}$ from observations of a superoutburst in 1994 June. This $P_{\mathrm{SH}}$ provides a superhump period excess $\varepsilon=\left(P_{\mathrm{SH}}-P_{\text {orb }}\right) / P_{\text {orb }}=0.033 \pm 0.009$. This value of the excess implies that TV Crv may be a peculiar object regarding its possibly large period excess compared with other short period systems (Patterson 2001). The error of $\varepsilon$ is, however, so large that the large $\varepsilon$ is not conclusive.

Here we report observations of four superoutbursts of TV Crv. Our observations on TV Crv provide new clues to understand the superhump period evolution related to the precursor phenomenon and the TTI model. In the next section, we mention our observation systems. In Sect. 3, we report detailed behaviour of superoutbursts and superhumps of TV Crv. We then discuss the implication of our results linked to the TTI model in Sects. 4 and 5. In Sect. 6, we compare and discuss our
Table 1. Journal of observations.

\begin{tabular}{cccrr}
\hline \hline ID & $T_{\text {start }}$ & $\delta T$ & $N$ & Site \\
\hline $01-01$ & 1957.1607 & 4.75 & 361 & Kyoto \\
$01-02$ & 1958.1373 & 5.28 & 243 & Tsukuba \\
$01-03$ & 1958.2718 & 2.12 & 176 & Kyoto \\
$01-04$ & 1959.0578 & 7.21 & 542 & Kyoto \\
$01-05$ & 1960.3133 & 0.75 & 64 & Kyoto \\
$01-06$ & 1961.1367 & 4.90 & 374 & Kyoto \\
$01-07$ & 1961.1470 & 3.04 & 70 & Tsukuba \\
$01-08$ & 1963.2215 & 1.96 & 138 & Kyoto \\
$01-09$ & 1964.0792 & 2.78 & 73 & Tsukuba \\
$01-10$ & 1965.1255 & 5.17 & 436 & Kyoto \\
$01-11$ & 1965.1483 & 3.81 & 94 & Tsukuba \\
$01-12$ & 1966.1710 & 0.56 & 39 & Kyoto \\
$01-13$ & 1969.1343 & 4.44 & 303 & Kyoto \\
$02-01$ & 2427.9767 & 2.18 & 132 & Kyoto \\
$02-02$ & 2428.0206 & 1.47 & 119 & Kyoto \\
$02-03$ & 2428.9583 & 2.23 & 195 & Kyoto \\
$02-04$ & 2428.9960 & 1.86 & 51 & Kyoto \\
$02-05$ & 2429.0071 & 1.56 & 147 & Okayama \\
$02-06$ & 2430.9581 & 1.16 & 103 & Kyoto \\
$02-07$ & 2430.9627 & 2.34 & 182 & Kyoto \\
$02-08$ & 2434.9773 & 2.03 & 161 & Kyoto \\
$03-01$ & 2769.9589 & 6.18 & 327 & Craigie \\
$03-02$ & 2776.9232 & 1.34 & 71 & Ellinbank \\
$03-03$ & 2777.0997 & 2.88 & 31 & Kyoto \\
$03-04$ & 2777.8617 & 2.18 & 111 & Ellinbank \\
$03-05$ & 2780.1247 & 1.35 & 37 & Kyoto \\
$03-06$ & 2781.0285 & 1.82 & 86 & Hida \\
$04-01$ & 3160.9673 & 2.46 & 237 & Kyoto \\
$04-02$ & 3161.9689 & 2.05 & 142 & Kyoto \\
$04-03$ & 3162.4693 & 5.84 & 210 & Concepción \\
$04-04$ & 3163.4698 & 6.18 & 238 & Concepción \\
$04-05$ & 3164.9970 & 0.70 & 34 & Barfold \\
$04-06$ & 3166.5550 & 3.81 & 182 & Concepción \\
$04-07$ & 3167.5709 & 3.40 & 195 & Concepción \\
$04-08$ & 3168.5882 & 2.86 & 176 & Concepción \\
$04-09$ & 3169.5472 & 1.67 & 57 & Concepción \\
$04-10$ & 3169.9654 & 2.63 & 123 & Kyoto \\
$04-11$ & 3170.9610 & 3.06 & 189 & Kyoto \\
\hline
\end{tabular}

$T_{\text {start }}=$ HJD-2 450000 .

$\delta T=$ Period of observations in hours.

$N=$ Number of images.

results with those for other known systems. Finally, we summarize our findings in Sect. 7.

\section{Observations}

We conducted observational campaigns for four superoutbursts of TV Crv which occurred in 2001 February-March, 2002 June, 2003 May, and 2004 July, through VSNET Collaboration (Kato et al. 2004b). Photometric observations were performed with unfiltered CCD cameras attached to 30-cm class telescopes at Concepción (2004), Kyoto (2001, 2002, 2003, and 2004), Tsukuba (2001), Okayama (2002), Craigie (2003), Ellinbank (2003), Hida (2003), and Barfold Observatory (2004). Our observation $\log$ is listed in Table 1. Each image was taken with an exposure time of $\sim 30 \mathrm{~s}$. After correcting for the standard de-biasing and flat fielding, we 
$2001 \mathrm{Feb} /$ Mar superoutburst

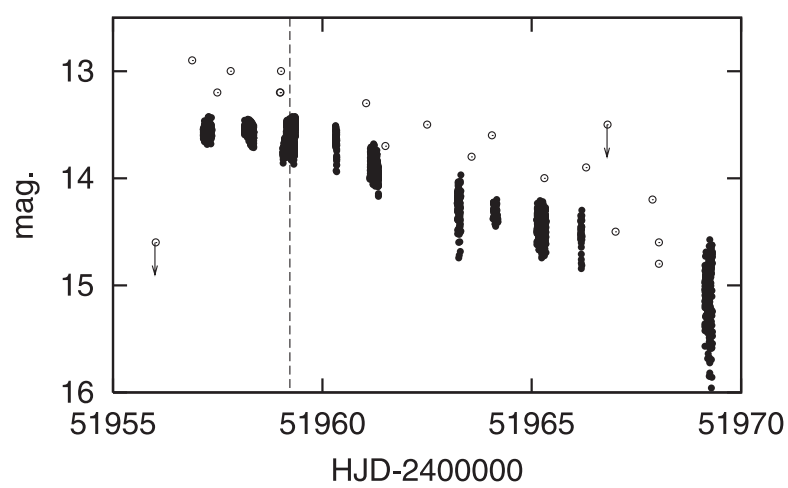

2004 Jun superoutburst

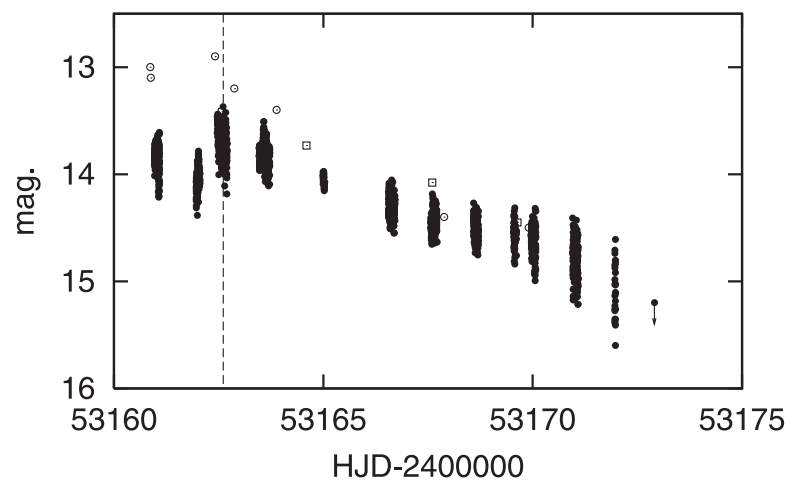

Fig. 1. Light curves of the 2001 February/March (left) and 2004 June (right) superoutbursts. The filled, open circles and open squares indicate our CCD observations, visual observations reported to VSNET, and observations by the ASAS-3 system, respectively (Pojmanski 2002). The vertical dashed lines in each panel show times when superhumps have the largest amplitude.

performed aperture and PSF photometry, then obtained differential magnitudes of the object using a neighbor comparison star UCAC2 24840990 (14.43 mag). The constancy of this comparison star was checked using another neighbor star UCAC2 24840985 (14.57 mag). In this paper, we neglect any small differences of magnitude systems between unfiltered CCD chips used by each observatory. Heliocentric time corrections were applied before the period analysis.

\section{Results}

Among the four superoutbursts, the evolution of superhumps was successfully detected even in early superoutburst phases during the 2001 and 2004 superoutbursts. On the other hand, the 2002 and 2003 superoutbursts were observed rather sparsely. We first report the former two superoutbursts focusing on their different features, and then shortly report the latter, poorly observed ones. Properties of all superoutbursts are summarized in Table 2. See the following sections for detailed information about the values in this table.

\subsection{The 2001 and 2004 superoutbursts}

The 2001 superoutburst was detected on February 18.392 (hereafter dates refer to UT) at a visual magnitude of 12.9. Visual observations reported to VSNET indicate that the object was fainter than 14.6 mag on February 17.517 and no preoutburst activity is seen before February 18 . The outburst was, hence, detected in a very early phase within one day just after the onset of the outburst. The first time-series CCD observation initiated on February 18.654, about $6 \mathrm{~h}$ after the visual detection.

The 2004 superoutburst was detected on June 4.362 (UT) at a visual magnitude of 13.0. Observations reported to VSNET indicate that it was fainter than 13.4 mag on May 28.399 (UT) and no pre-outburst activity is seen before June 4 . The first time-series observation initiated on June 4.463 (UT), about 2 h after the visual detection.

The light curves of the superoutbursts in February/March 2001 and June 2004 are shown in Fig. 1. The most noteworthy point in the light curves is their different behaviour during the first few days. While the light curve in 2001 is described with a monotonic fading, the light curve in 2004 shows a 0.4 mag rebrightening $1.7 \mathrm{~d}$ after the outburst detection. This observation reveals that the early outburst was actually a precursor of the late genuine supermaximum. In conjunction with the close monitoring of the object, we conclude that no precursor event was associated with the 2001 superoutburst.

We succeeded in obtaining time-series data during the early phase of the superoutbursts, which are shown in Fig. 2. We also show the observation IDs (see Table 1) and typical errors in each panel. As can be seen in Fig. 2, no superhump-like modulation appears except for the "04-02", in which a 0.3-mag hump is detected. The "04-02" run lasted $2.05 \mathrm{hr}$ which well covers an orbital period of TV Crv. Throughout this run, the object is on a rapid brightening trend at a rate of $2.6 \mathrm{mag} \mathrm{d}^{-1}$. The hump is superimposed on this brightening trend. This indicates that the temporary fading from the precursor had already been terminated, and then started brightening to the supermaximum during the "04-02" run.

The other panels of the "01-01", "01-02", and "04-01" in Fig. 2 show modulations with rather small amplitudes $(\sim 0.1 \mathrm{mag})$ and long timescales. No periodic signal is detected in these runs with our Fourier analysis in the period range of $10 \mathrm{~s}-0.1 \mathrm{~d}$. On the other hand, we note that possible $0.1-$ 0.2 mag amplitude short-term fluctuations with timescale of $\sim 10$ min can be seen in the "01-02" run.

We detected superhumps after this early phase. In the case of the 2001 superoutburst, fully grown superhumps appeared on JD 2451959 (the "01-04" run). In the case of the 2004 superoutburst, on the other hand, the supermaximum coincides with the apparition of superhumps with the largest amplitude of $~ 0.4$ mag (the "04-03" run).

A period analysis with the PDM method (Stellingwerf 1978) was performed after linear trends were subtracted from the light curves. We used light curves between JD 2451959.0 and 2451966.2 for the 2001 superoutburst and between JD 2453162.4 and 2453171.1 for the 2004 superoutburst. The samples for the 2001 and 2004 superoutbursts contain 1830 and 1692 photometric points, respectively. The PDM analysis 
Table 2. Observational properties of superoutbursts.

\begin{tabular}{lcccc}
\hline \hline & 2001 & 2002 & 2003 & 2004 \\
\hline Precursor & No & No? & No? & Yes \\
$P_{\mathrm{SH}}($ day) & $0.065028(0.000008)$ & $0.064981(0.000053)$ & $0.0674(0.0024)$ & $0.065023(0.000013)$ \\
$\dot{P}_{\mathrm{SH}} / P_{\mathrm{SH}}\left(10^{-5}\right)$ & $7.96(0.73)$ & - & - & $-0.32(1.20)$ \\
Fading rate $\left(\mathrm{mag} \mathrm{d}^{-1}\right)$ & $0.12(0.01)$ & $0.17(0.02)$ & $0.17(0.01)$ & $0.13(0.01)$ \\
Duration (day) & 12 & 10 & 12 & 12 \\
Time interval from the last & - & 468 & 345 & 392 \\
superoutburst (day) & & & & \\
\hline
\end{tabular}
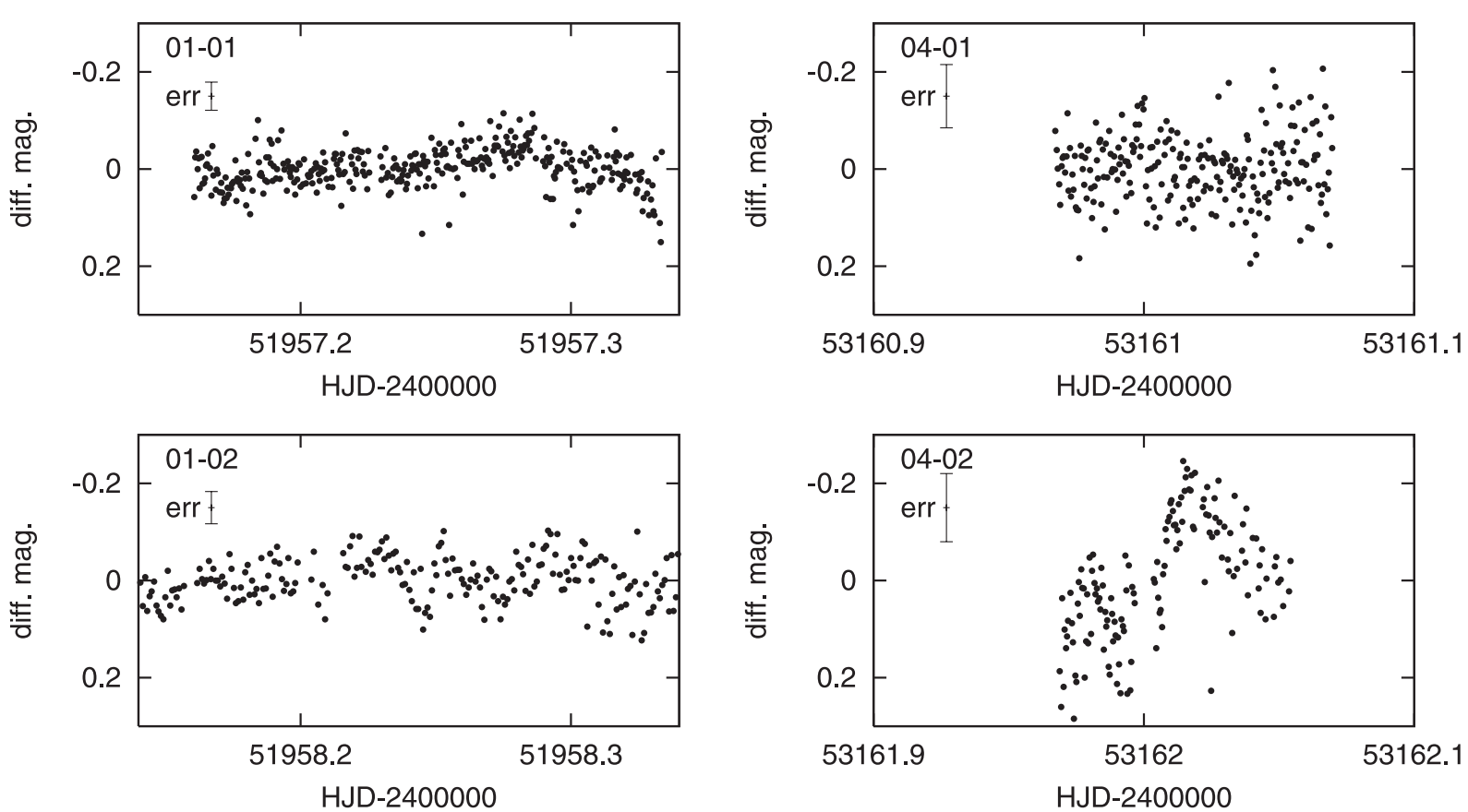

Fig. 2. Light curves during early phases of superoutbursts in 2001 and 2004 . The abscissa and ordinate denote the time in HJD and the differential magnitudes, respectively. The magnitude system is normalized by subtracting average magnitudes of each panel. We indicate the run ID number (Table 1) and typical errors in each panel.
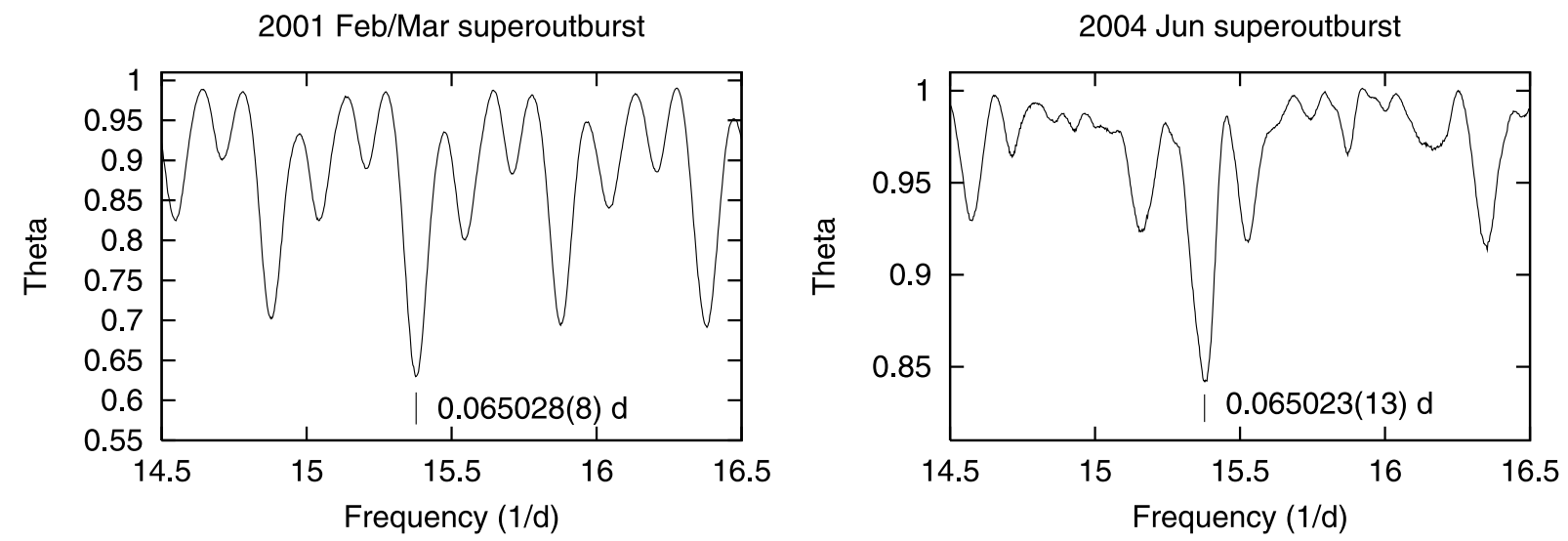

Fig. 3. Frequency- $\Theta$ diagrams for the 2001 (left) and 2004 (right) superoutbursts calculated by the PDM method.

yielded the frequency- $\Theta$ diagram shown in Fig. 3. The superhump periods are calculated to be $0.065028 \pm 0.000008 \mathrm{~d}$ (2001) and $0.065023 \pm 0.000013 \mathrm{~d}$ (2004). These are in agreement each other and also in agreement with $P_{\mathrm{SH}}$ reported in Howell et al. (1996b) $(0.0650 \pm 0.0008 \mathrm{~d})$. Since the error of $P_{\mathrm{SH}}$ is smaller in 2001 than that in 2004, we adopt $P_{\mathrm{SH}}$ of
TV Crv to be $0.065028 \pm 0.000008 \mathrm{~d}$ in this paper. According to Woudt \& Warner (2003), the orbital period of TV Crv is $0.06288 \pm 0.00013 \mathrm{~d}$, which yields a superhump period excess $\varepsilon=0.0342 \pm 0.0021$. The $3.4 \%$ superhump excess is relatively large for short-period SU UMa systems (Patterson et al. 2003). 


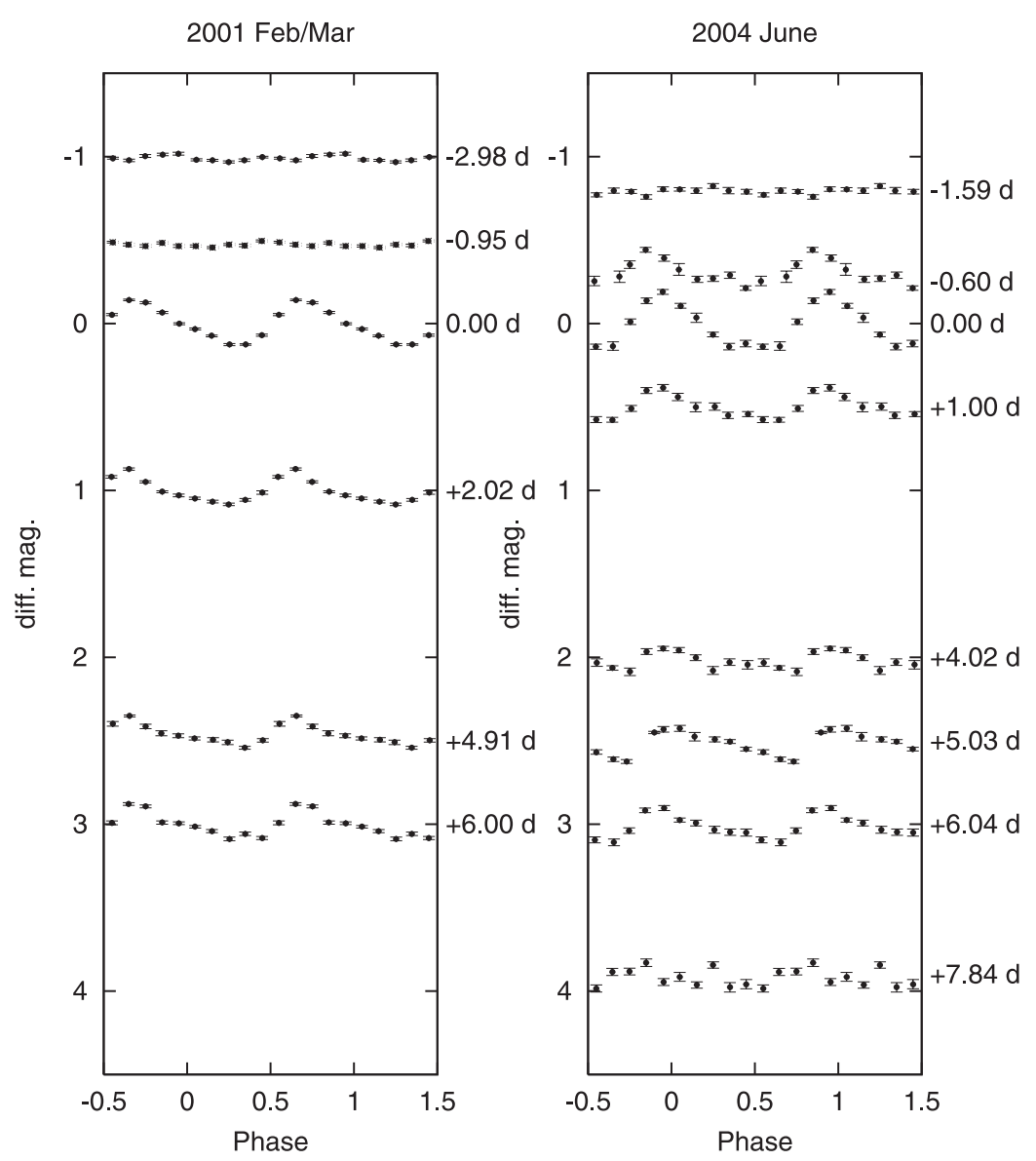

Fig. 4. Superhump evolution during the 2001 (left) and 2004 (right) superoutbursts. The abscissa and ordinate denote the superhump phase and the differential magnitude, respectively. The phase is calculated with a superhump period of $0.065028 \mathrm{~d}$ and an arbitrary epoch. The differential magnitudes are normalized by each average magnitude, and are sorted with observation times which are indicated on the right vertical axis of each panel. See the text for detailed information.

Figure 4 shows the evolution of the superhumps from the early phase including the precursor to the end of the superoutburst plateau. All light curves are folded with $P_{\mathrm{SH}}=$ $0.065028 \mathrm{~d}$ and an arbitrary epoch. The abscissa and ordinate denote the phase and the differential magnitude, respectively. We calculated center times of each run and show them in the figure. We set the origin of the times at the "0104" and "04-03" runs, in which superhumps had the largest amplitude. The differential magnitudes are normalized by each average magnitude, and are shifted by constants proportional to the times of each run in order to clearly compare two sequences. The hump just before the supermaximum on 2004 has a peak phase roughly the same as those of later superhumps. It strongly indicates that the hump is actually a superhump, growing to the supermaximum, as observed in T Leo (Kato 1997), V436 Cen (Semeniuk 1980), and GO Com (Imada et al. 2004). As can be seen from both panels, the amplitude of superhumps decreased in a few days, then kept 0.2-mag peak-to-peak amplitudes for 6 days. The 2001 and 2004 superoutbursts, thus, have quite similar characteristics regarding the evolution of superhump amplitudes.

We determined peak times of superhumps by taking crosscorrelation between the light curve and average profiles of superhumps. With determined peaks and $P_{\mathrm{SH}}$ of $0.065028 \mathrm{~d}$, we calculate the $\mathrm{O}-\mathrm{C}$ of the superhump maximum timings, which is shown in Fig. 5. There is an obvious difference between the $\mathrm{O}-\mathrm{C}$ in the 2001 and 2004 superoutbursts. The $\mathrm{O}-\mathrm{C}$ clearly indicates an increase of $P_{\mathrm{SH}}$ with time in the case of the 2001 superoutburst. A quadratic fit to the $\mathrm{O}-\mathrm{C}$ yields a period derivative of $\dot{P}_{\mathrm{SH}} / P_{\mathrm{SH}}=7.96 \pm 0.73 \times 10^{-5}$. On the other hand, the $\mathrm{O}-\mathrm{C}$ is almost constant, in other words, $P_{\mathrm{SH}}$ was stable during the 2004 superoutburst. A quadratic fit yields $\dot{P}_{\mathrm{SH}} / P_{\mathrm{SH}}=-0.32 \pm 1.20 \times 10^{-6}$. This result indicates that the superhumps in 2004 superoutburst have quite small $\dot{P}_{\mathrm{SH}} / P_{\mathrm{SH}}$ compared with other systems (Kato et al. 2003c).

We note that there is a slight phase shift at the hump just before the supermaximum in 2004 superoutburst, as shown in the right panel of Fig. 5. The slight phase shift in the early stage implies that superhumps evolved with a rapid period change just before the supermaximum. Similar rapid period changes during very early phases are also known in T Leo (Kato 1997), V1028 Cyg (Baba et al. 2000), and XZ Eri (Uemura et al. 2004).

\subsection{The 2002 superoutburst}

The 2002 superoutburst was first detected on May 30.399 (UT) at a visual magnitude of $13.1 \mathrm{mag}$. The ASAS-3 system records 

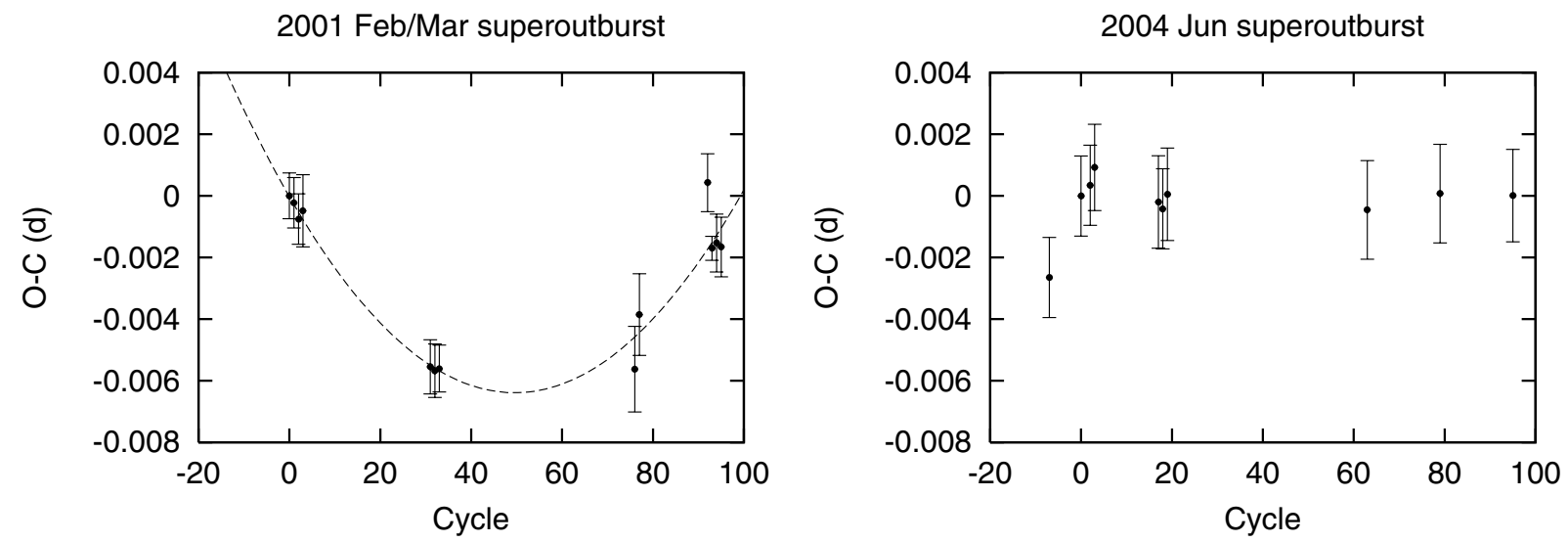

Fig. 5. O-C diagrams of superhumps during the 2001 (left) and 2004 (right) superoutbursts. The abscissa and ordinate denote the cycle and the $\mathrm{O}-\mathrm{C}$ in day, respectively. The dashed line in the left panel is the best fitted quadratic curve for the O-C in 2001.

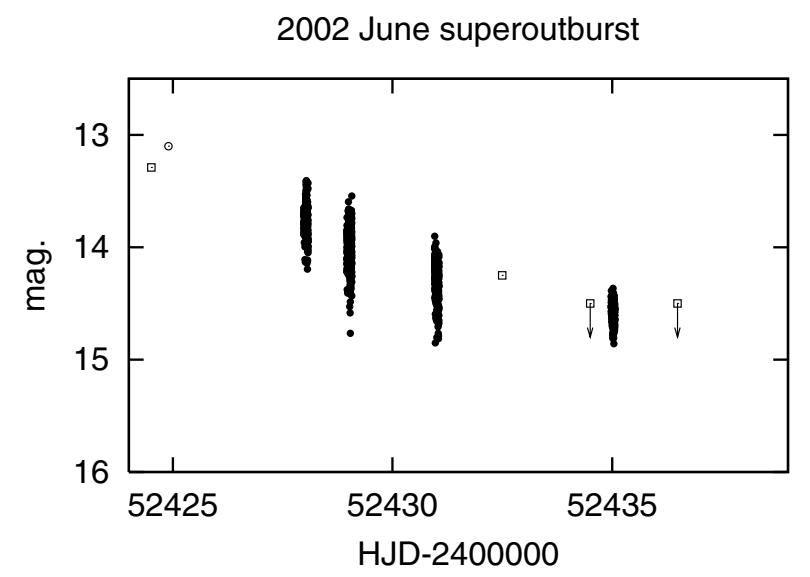

Fig. 6. Light curve of the superoutburst in 2002 June. The symbols are the same as in Fig. 1.

an earlier detection of the outburst on May 30.009 (UT) and a negative detection on May 21.048 (UT) (Pojmanski 2002). Unfortunately, there is no time-series data just after the outburst detection. The first run (the "02-01" run in Table 1) initiated at June 2.476 (UT). The light curve of the superoutburst is shown in Fig. 6. The "02-01" run detected superhumps, which establish that this outburst is a superoutburst. Profiles of superhumps during this superoutburst are shown in Fig. 7. Figure 8 is the $\mathrm{O}-\mathrm{C}$ diagram of superhumps. While it contains only three points, this figure apparently implies a period increase of superhumps, as observed in the 2001 superoutburst.

\subsection{The 2003 superoutburst}

The 2003 superoutburst was discovered by a visual observation on May 9.546 (UT) at $13.1 \mathrm{mag}$. The latest negative visual observation had been reported on May 6.412 (UT) (fainter than $14.6 \mathrm{mag}$ ), three days before the outburst detection. The first time-series observation initiated at May 10.458 (UT), about one day after the outburst detection. Considering the rapid evolution during the precursor in the 2001 superoutburst, we cannot exclude the possibility that the 2003 superoutburst had a precursor between May 6 and 9. The light curve of this outburst is shown in Fig. 9. The first run "03-01" clearly detects

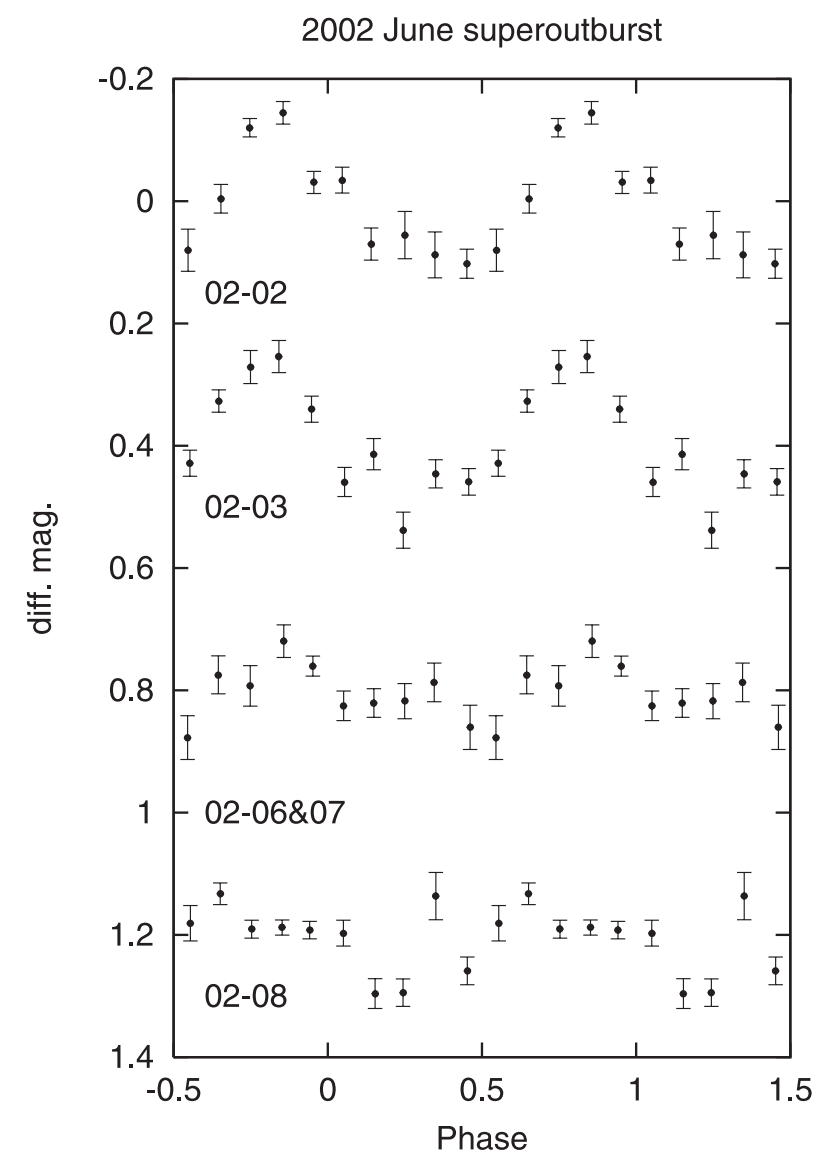

Fig. 7. Superhump evolution during the 2002 superoutburst. The symbols in the figure are the same as in Fig. 4.

fully grown superhumps, as shown in Fig. 10, which reveal that it is another superoutburst. Due to the lack of enough observations, we cannot find any hints of significant period changes of superhumps.

\section{Implication for the TTI model}

The observational properties of the four superoutbursts are summarized in Table 2. TV Crv is one of the typical short orbital period SU UMa-type dwarf novae. Its supercycle is 


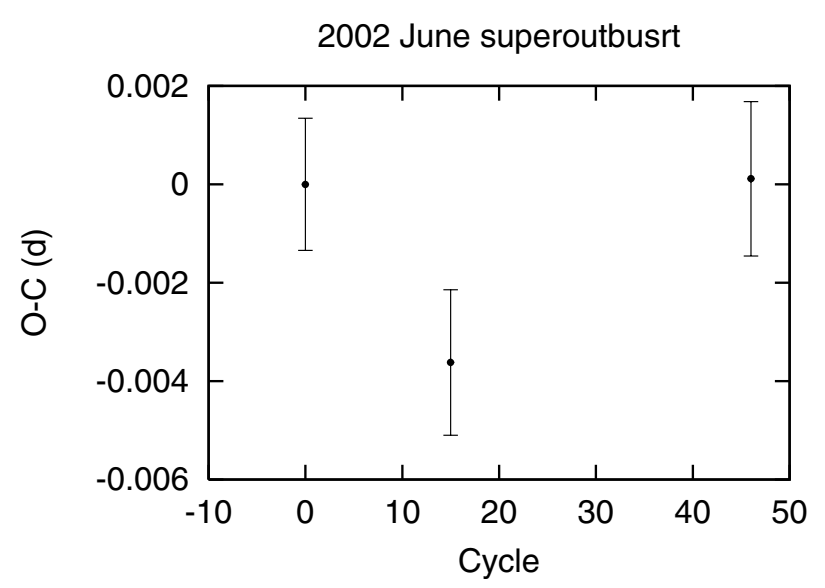

Fig. 8. O-C diagram of superhumps during the 2002 superoutburst. The symbols in the figure are the same as in Fig. 5.

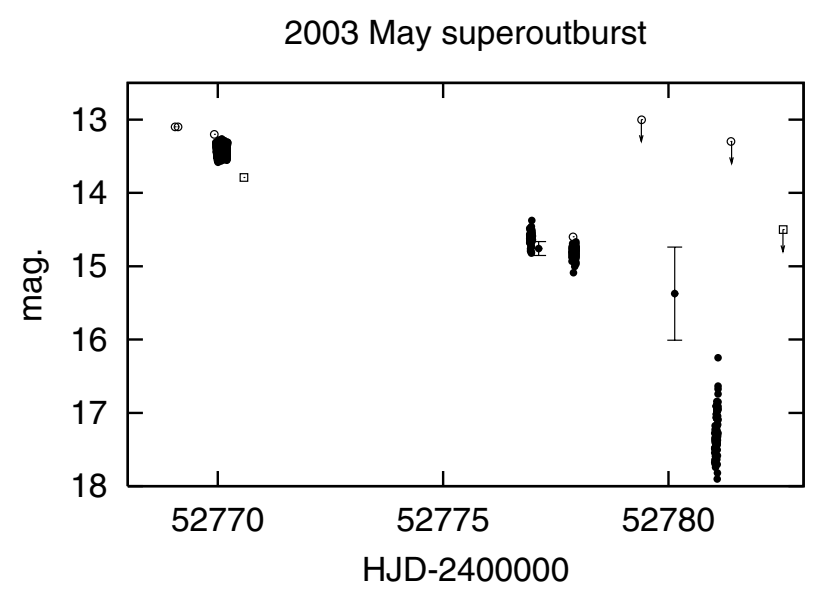

Fig. 9. Light curve of the superoutburst in 2003 May. The symbols in the figure are the same as in Fig. 1.

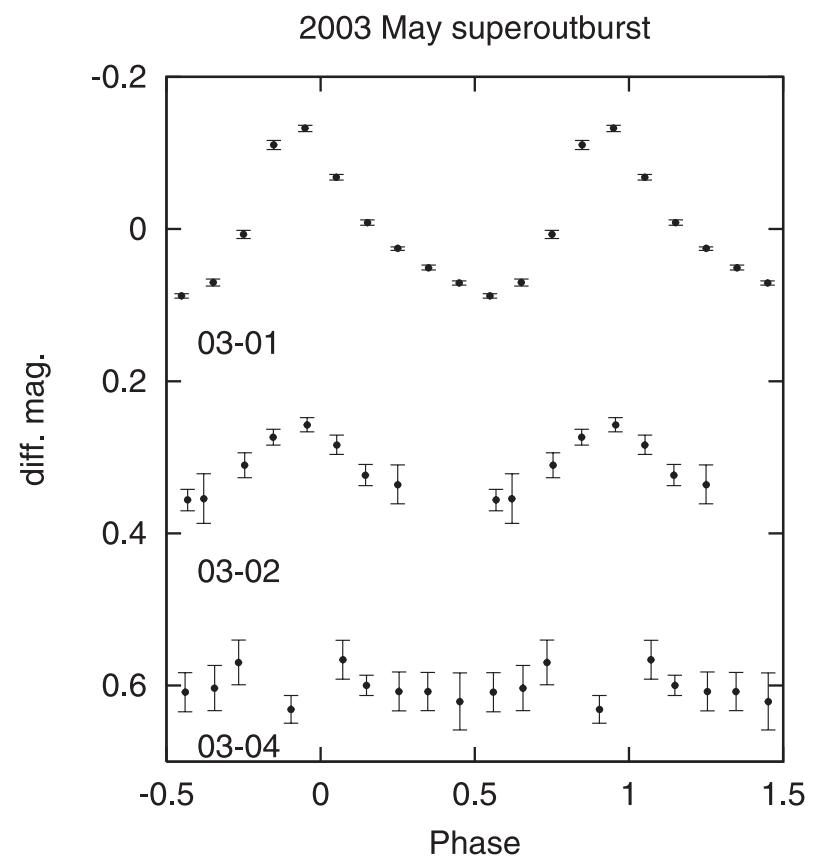

Fig. 10. Superhump evolution during the 2003 superoutburst. The symbols in the figure are the same as in Fig. 4. calculated to be $402 \pm 51 \mathrm{~d}$ from the three time-intervals of superoutbursts listed in Table 2. This supercycle is also a typical value for SU UMa stars. A noteworthy feature of TV Crv is its superhump excess (3.4\%), which is relatively large for shortperiod systems, but not extraordinary (Patterson et al. 2003). It is well known that the superhump period excess is related to the superhump period (Patterson et al. 2003). From the theoretical point of view, this can be understood since the precession velocity of the eccentric disk depends on the disk radius and the mass ratio of binary systems. The superhump period excess, $\varepsilon$, can be expressed as (Osaki 1985);

$\varepsilon=\frac{3}{4} \frac{q}{\sqrt{1+q}}\left(\frac{r_{\mathrm{d}}}{a}\right)^{3 / 2}$.

Assuming a certain disk radius at which the tidal mode is excited, one can describe the superhump period excess as a function of the superhump period (Mineshige et al. 1992). The large superhump excess of TV Crv, therefore, implies a relatively large mass ratio among short-period SU UMa stars. The empirical relationship in Patterson (2001) yields the mass ratio to be $q=0.16 \pm 0.01$ for TV Crv. On the other hand, it is possible that the large superhump excess is partly caused by an unusually large disk radius. The large mass ratio of TV Crv should be confirmed by spectroscopic observations in future.

Our observations reveal that TV Crv experiences two types of superoutbursts, that is, one with a precursor and the other without a precursor. Similar morphology studies of superoutburst light curves had been performed for VW Hyi, which also shows the two types of superoutbursts (Bateson 1977; Marino \& Walker 1979). VW Hyi is a typical SU UMa-type dwarf novae having a relatively long orbital period of $0.074271 \mathrm{~d}$ (Downes et al. 2001). Our observations of TV Crv are the first to show that those two types of superoutbursts appear even in short orbital period systems.

To explain the behaviour of VW Hyi, Osaki \& Meyer (2003) propose the refined TTI model, in which the types of superoutburst depend on the maximum radius of the accretion disk. When the accretion disk reaches the tidal truncation radius, the dammed matter prevents the disk from a propagation of a cooling wave, leading to a superoutburst without a precursor. In this view, a large mass ratio is required for a system to achieve the situation that the tidal truncation radius lies just beyond the 3:1 resonance radius. On the other hand, when the disk fails to reach the tidal truncation radius, a rapid fading initiates. This fading is terminated, and the object rebrightens to a supermaximum due to a growth of the tidal dissipation. In this case, a large mass ratio is also required for a rapid growth of the tidal dissipation before the object returns to quiescence. VW Hyi has a superhump excess of $3.9 \%$ (van Amerongen et al. 1987), which yields a mass ratio $q=0.18$ from the empirical relationship in Patterson (2001). Tappert et al. (2003) reported $q \sim 0.14$ for VW Hyi based on their spectroscopic observations. The mass ratio of TV Crv is possibly close to that of VW Hyi rather than those of ordinary short period SU UMa stars (Patterson 2001).

Although TV Crv is a short period system, we propose that it has a relatively large mass ratio. According to Osaki \& Meyer (2003), a system having a large mass ratio 
$(q \sim 0.2)$ can experience the two types of superoutburst. The behaviour of TV Crv can, therefore, be explained by the refined TTI model, furthermore, it possibly provides evidence that the mass ratio plays a key role in the morphology of superoutburst light curve.

\section{Presence of a precursor and superhump evolution}

The most important and unforeseen finding in our observation is that the $\dot{P}_{\mathrm{SH}} / P_{\mathrm{SH}}$ can be variable in distinct superoutbursts in one system. This is clearly shown in Table 2; a positive $\dot{P}_{\mathrm{SH}} / P_{\mathrm{SH}}$ in the 2001 superoutburst and an almost constant $P_{\mathrm{SH}}$ in the 2004 superoutburst. Except for the difference in $\dot{P}_{\mathrm{SH}} / P_{\mathrm{SH}}$, another observational difference between these two superoutbursts is the presence or absence of the precursor. There was no precursor in the 2001 superoutburst, while a clear precursor was observed in 2004. Our observation hence indicates that the $\dot{P}_{\mathrm{SH}} / P_{\mathrm{SH}}$ is related to the precursor phenomenon.

As mentioned above, the TTI model suggests that the appearance of the precursor depends on whether the disk reaches the tidal truncation radius or not. Based on this idea, at the time when superhumps are fully grown, the disk size should be different in the two types of superoutbursts. In the case of the precursor-main type outburst, the disk size is around the 3:1 resonance radius at supermaximum. On the other hand, in the case of the superoutburst without a precursor, the hot disk can remain larger than the 3:1 resonance radius due to the dammed matter at the tidal truncation radius. The accretion disk can, hence, have a relatively large amount of gas beyond the 3:1 resonance radius even a few days after the supermaximum when superhumps are fully grown. We therefore propose that the $\dot{P}_{\mathrm{SH}} / P_{\mathrm{SH}}$ is related to the amount of the gas around and beyond the 3:1 resonance radius.

We now present an idea how the disk size actually affects the eccentric disk evolution. We first consider the standard picture of the eccentric disk evolution. In an early phase of outburst, the rapid excitation of the eccentric mode stops when the angular momentum removal by the tidal dissipation is balanced with the input angular momentum transfered from the inner region. In the case of the precursor-main type outburst, then, the accretion disk shrinks below the 3:1 resonance radius at that time (Whitehurst 1994). The eccentricity wave can only propagate inward, since the tidal mode is no longer excited. In the case of the superoutburst without a precursor, on the other hand, we can expect a large amount of gas over the 3:1 resonance radius at that time. We conjecture that the eccentric mode can keep excited because the disk radius presumably remains larger than the 3:1 resonance radius. The positive $\dot{P}_{\mathrm{SH}} / P_{\mathrm{SH}}$ can be explained by a gradual outward propagation of the eccentricity wave.

It is, however, unclear whether the outward propagation is possible only with the large disk. The outward propagation essentially requires an additional input of angular momentum from an inner region. It might be possible that the gas in an inner region may be swept up, then give additional angular momentum into the outermost area of the eccentricity wave. This additional supply of angular momentum would enable to keep the disk size large and the continuous excitation of the eccentric mode.

Olech et al. (2003) propose that the $\dot{P}_{\mathrm{SH}} / P_{\mathrm{SH}}$ is negative at the beginning and the end of the superoutburst, but positive in the middle phase for several SU UMa-type dwarf novae. Based on our scenario, the duration of the positive $\dot{P}_{\mathrm{SH}} / P_{\mathrm{SH}}$ depends on the amount of the gas which enables the continuous excitation of the eccentric mode. The transition from a positive $\dot{P}_{\mathrm{SH}} / P_{\mathrm{SH}}$ to a negative one may be explained by the depletion of the gas.

The above discussion is summarized in the following two ideas: i) At the time when superhumps are fully grown, the accretion disk remains larger in the superoutburst without the precursor than in the precursor-main type superoutburst. ii) Even after that, the eccentric mode keeps excited through a superoutburst. These ideas should be tested by hydrodynamical simulations.

\section{Discussion}

We revealed that the $\dot{P}_{\mathrm{SH}} / P_{\mathrm{SH}}$ is variable in distinct superoutbursts for TV Corvi. This result should be confirmed by observations of other sources in future because we now have no data of variations of the $\dot{P}_{\mathrm{SH}} / P_{\mathrm{SH}}$ against different types of superoutburst in other sources. On the other hand, it is valuable to investigate the relationship of $\dot{P}_{\mathrm{SH}} / P_{\mathrm{SH}}$ and the type of superoutburst in known systems. To perform this, we collected the sample of 40 dwarf novae and one X-ray binary whose $\dot{P}_{\mathrm{SH}} / P_{\mathrm{SH}}$ is published, as listed in Table 3 . We now classify the morphology of superoutburst light curve into two types, that is, the type " $A$ " and type " $B$ ". The type A is defined by the detection of a precursor, in other words, the precursor-main type superoutburst. On the other hand, the type B is defined by the detection of a delay of the superhump growth after a supermaximum. In our sample listed in Table 3, we find 6 and 7 cases for the type A and B, respectively. There is no system having both features. WZ Sge-type dwarf novae are indicated by "WZ" in Table 3 because their superhump evolution is peculiar; they have an early hump era followed by an ordinary superhump era (Kato et al. 1996). The sample in Table 3 has 8 cases for 5 WZ Sge stars. Types of superoutburst are unclear in the other 29 cases due to the lack of enough observations during early phases of superoutbursts. The $\dot{P}_{\mathrm{SH}} / P_{\mathrm{SH}}$ are shown against $P_{\mathrm{SH}}$ in Fig. 11.

As mentioned above, WZ Sge-type systems tend to show positive $\dot{P}_{\mathrm{SH}} / P_{\mathrm{SH}}$ as indicated by filled squares in Fig. 11 . In these systems, their long recurrence time and the lack of normal outburst lead to a huge amount of accumulated gas compared with ordinary SU UMa systems. At the onset of their outburst, the accretion disk, hence, violently expands beyond the 3:1 resonance radius. The large disk in WZ Sge stars may partly be due to a continuous expansion of their quiescent disks, as proposed in Mineshige et al. (1998). This situation in WZ Sge systems is similar to the type B outburst in TV Crv discussed in the last section. Kato et al. (2004a) propose a scenario analogous to that described in the last section for positive $\dot{P}_{\mathrm{SH}} / P_{\mathrm{SH}}$ in WZ Sge-type dwarf novae. The difference between WZ Sge systems and TV Crv is the mechanism to generate a large disk 
Table 3. Superhump period derivative and the type of superoutbursts.

\begin{tabular}{llccc}
\hline \hline Object & $P_{\text {SH }}$ & $\dot{P}_{\text {SH }} / P_{\text {SH }}$ & Type & Ref. \\
& (day) & $\left(10^{-5}\right)$ & & \\
\hline V485 Cen & 0.04216 & $28(3)$ & - & 1 \\
EI Psc & 0.04627 & $12(2)$ & - & 2 \\
WZ Sge(1978) & 0.05722 & $-1(4)$ & WZ & 3 \\
WZ Sge(2001) & 0.05719 & $0.1(0.8)$ & WZ & 4 \\
AL Com(1995) & 0.0572 & $2.1(0.3)$ & WZ & 5 \\
HV Vir & 0.05820 & $5.7(0.6)$ & WZ & 6 \\
HV Vir(2002) & 0.05826 & $7.8(7)$ & WZ & 7 \\
SW UMa(1991) & 0.0583 & $6(4)$ & - & 6 \\
SW UMa(1996) & 0.0583 & $4.4(0.4)$ & B & 8 \\
WX Cet(1996) & 0.0593 & $4(2)$ & - & 6 \\
WX Cet(1998) & 0.05949 & $8.5(1.0)$ & B & 9 \\
T Leo & 0.0602 & $-0.5(0.3)$ & A & 10 \\
EG Cnc & 0.06038 & $2.0(0.4)$ & WZ & 11 \\
EG Cnc & 0.06043 & $1.7(1)$ & WZ & 12 \\
GO Com & 0.06306 & $18(3)$ & A & 13 \\
V1028 Cyg & 0.06154 & $8.7(0.9)$ & B & 14 \\
XZ Eri & 0.06281 & $-1.4(0.2)$ & - & 15 \\
V1159 Ori & 0.0642 & $-3.2(1)$ & A & 16 \\
VY Aqr & 0.0644 & $-8(2)$ & - & 17 \\
OY Car & 0.06443 & $-5(2)$ & - & 18 \\
TV Crv(2001) & 0.06503 & $8.0(0.7)$ & B & 19 \\
TV Crv(2004) & 0.06502 & $-0.03(0.12)$ & A & 19 \\
UV Per & 0.06641 & $-2.0(1)$ & - & 6 \\
CT Hya & 0.06643 & $-2(8)$ & - & 20 \\
DM Lyr & 0.06709 & $5.7(17.2)$ & - & 21 \\
SX LMi & 0.0685 & $-8(2)$ & - & 22 \\
KS UMa(2003 early) & 0.07009 & $-21(8)$ & - & 23 \\
KS UMa(2003 late) & 0.07009 & $21(12)$ & - & 23 \\
RZ Sge(1994) & 0.07042 & $-10(2)$ & - & 24 \\
RZ Sge(1996) & 0.07039 & $-11.5(1)$ & - & 25 \\
CY UMa & 0.0724 & $-5.8(1.4)$ & - & 26 \\
VW Crb & 0.07287 & $9.3(0.9)$ & - & 27 \\
NSV 10934 & 0.07485 & $-10.2(1.0)$ & - & 28 \\
CC Cnc & 0.07552 & $-10.2(1.3)$ & - & 29 \\
V1251 Cyg & 0.07604 & $-12(4)$ & B & 30 \\
QW Ser(2000) & 0.07698 & $-4.2(0.8)$ & - & 31 \\
QW Ser(2002) & 0.07697 & $-7.3(3.1)$ & - & 31 \\
VW Hyi & 0.07714 & $-6.5(0.6)$ & - & 32 \\
Z Cha & 0.07740 & $-4(2)$ & A & 33 \\
TT Boo(2004 early) & 0.07796 & $-52.3(1.3)$ & B & 34 \\
TT Boo(2004 middle) & 0.07796 & $12.3(4.8)$ & B & 34 \\
\hline & 0.07796 & $-6.2(0.9)$ & B & 34 \\
\hline
\end{tabular}

over the 3:1 resonance radius. In the case of WZ Sge systems, Osaki \& Meyer (2003) propose that the large disk is maintained by the strong tidal removal of angular momentum at the $2: 1$ resonance radius. The disk can reach the $2: 1$ resonance radius because of the large amount of accumulated matter. In the case of the type B outbursts of TV Crv, the large disk is maintained at the tidal truncation radius. This is due to a high mass ratio
Table 3. continued.

\begin{tabular}{llccc}
\hline \hline Object & $\begin{array}{l}P_{\mathrm{SH}} \\
(\text { day })\end{array}$ & $\begin{array}{c}\dot{P}_{\mathrm{SH}} / P_{\mathrm{SH}} \\
\left(10^{-5}\right)\end{array}$ & Type & Ref. \\
\hline RZ Leo & 0.07853 & $5.9(1.0)$ & $\mathrm{WZ}$ & 35 \\
SU UMa & 0.0788 & $-10(3)$ & - & 36 \\
HS Vir & 0.08077 & $-4(1)$ & - & 37 \\
V877 Ara & 0.08411 & $-14.5(2.1)$ & - & 38 \\
EF Peg(1991) & 0.0871 & $-2.2(1)$ & $\mathrm{B}$ & 39 \\
BF Ara & 0.08797 & $-0.8(1.4)$ & - & 40 \\
KK Tel & 0.08801 & $-37(4)$ & - & 7 \\
V344 Lyr & 0.09145 & $-0.8(0.4)$ & - & 41 \\
YZ Cnc & 0.09204 & $-7(2)$ & - & 42 \\
V725 Aql & 0.09909 & $\sim 0$ & - & 43 \\
MN Dra & 0.10768 & $-170(2)$ & - & 44 \\
TU Men & 0.1262 & $-9(2)$ & - & 45 \\
XTE J1118+480 & 0.17053 & $-0.6(0.1)$ & A & 46 \\
\hline
\end{tabular}

References: 1. Olech (1997), 2. Uemura et al. (2002a), 3. Kuulkers et al. (2002), 4. Ishioka et al. (2002), 5. Nogami et al. (1997a), 6. Kato et al. (2001b), 7. Ishioka et al. (2003), 8. Nogami et al. (1998), 9. Kato et al. (2001a), 10. Kato (1997), 11. Kato et al. (1997), 12. Kato et al. (2004a), 13. Imada et al. (2004), 14. Baba et al. (2000), 15. Uemura et al. (2004), 16. Patterson et al. (1995), 17. Patterson et al. (1993), 18. Schoembs (1986), 19. this work, 20. Kato et al. (1999), 21. Nogami et al. (2003a), 22. Nogami et al. (1997b), 23. Olech et al. (2003), 24. Kato (1996), 25. Semeniuk et al. (1997), 26. Harvey \& Patterson (1995), 27. Nogami et al. (2004b), 28. Kato et al. (2003b), 29. Kato et al. (2002), 30. Kato (1995), 31. Nogami et al. (2004a), 32. Haefner et al. (1979), 33. Kuulkers et al. (1991), 34. Olech et al. (2004), 35. Ishioka et al. (2001), 36. Udalski (1990), 37. Kato et al. (1998), 38. Kato et al. (2003c), 39. Kato (2002), 40. Kato et al. (2003a), 41. Kato (1993), 42. Patterson (1979), 43. Uemura et al. (2001), 44. Nogami et al. (2003b), 45. Stolz \& Schoembs (1984), 46. Uemura et al. (2002c).

leading to the tidal truncation radius just beyond the $3: 1$ resonance radius.

We can therefore consider that a similar physical condition appears in the type B superoutbursts and the WZ Sge-type superoutbursts, in terms of the superhump evolution. In Fig. 11, we show these objects as filled symbols (squares for WZ Sge stars and circles for the type B) and the type A superoutburst as open circles. We can see a tendency that the B- and WZ-types generally have larger $\dot{P}_{\mathrm{SH}} / P_{\mathrm{SH}}$, as expected from our scenario. This figure, however, also show the presence of two exceptions breaking the tendency (GO Com and V1251 Cyg). The nature of these objects is an open issue. We need to obtain their $\dot{P}_{\mathrm{SH}} / P_{\mathrm{SH}}$ in another superoutburst to investigate their possible variations.

The two systems having the shortest $P_{\mathrm{SH}}$ in Fig. 11 are V485 Cen and EI Psc. While they have ultra-short orbital periods, their secondaries are relatively massive (Augusteijn et al. 1993; Thorstensen et al. 2002). The superhump period excess and mass ratio of EI Psc are $\varepsilon=0.040$ and $q=0.19$, respectively, which are actually larger than those of TV Crv and VW Hyi (Uemura et al. 2002b). According to the refined TTI model, their accretion disks can reach the tidal truncation radius and remain active in the eccentric mode through a 


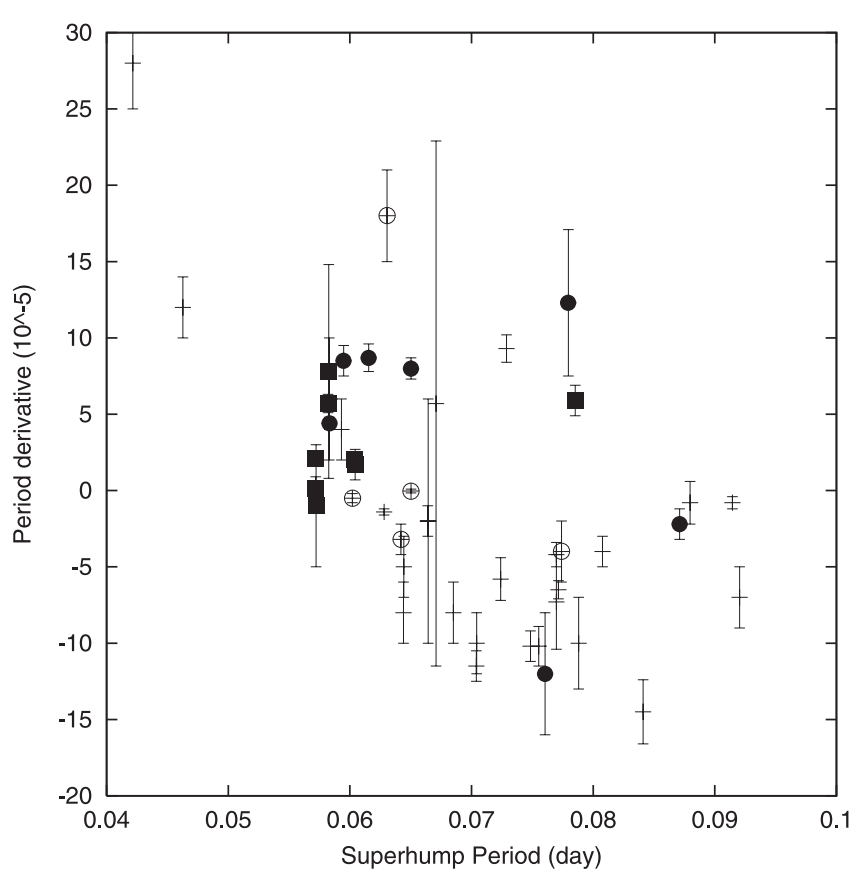

Fig. 11. The superhump period derivative against the superhump period for the SU UMa-type dwarf novae listed in Table 3. The open circles indicate type A superoutbursts which have a precursor. The filled circles indicate type B superoutbursts in which a delay of superhump growth is observed. The filled squares indicate WZ Sge-type dwarf novae. The other points indicated by the crosses are objects whose outburst types are unknown. The figure focuses on objects whose outburst types are known. We, hence, omit three unknown-type dwarf novae, KK Tel, MN Dra, (exceptionally large period derivatives) and TU Men (a long superhump period) in this figure. We only show positive values of period derivatives for KS UMa and TT Boo, in which changes of the period derivative have been observed.

superoutburst. Their high $\dot{P}_{\mathrm{SH}} / P_{\mathrm{SH}}$ can, hence, be naturally explained with our scenario. Observations of the onset of their superoutbursts are encouraged to reveal the type of them.

The only X-ray binary in Table 3, XTE J1118+480, is a black hole X-ray binary (BHXB) having a quite low mass ratio $q=0.05$ (Wagner et al. 2001). This is a unique object in the point that the $\dot{P}_{\mathrm{SH}} / P_{\mathrm{SH}}$ is significantly determined in BHXBs. Although the low mass ratio implies a situation similar to WZ Sge-type dwarf novae, its $\dot{P}_{\mathrm{SH}} / P_{\mathrm{SH}}$ is slightly, but significantly negative as listed in Table 3 . On the other hand, its main outburst has a precursor, which is reminiscent of the precursormain type superoutburst in SU UMa systems (Kuulkers 2001). The accretion disk radius was probably just around the 3:1 resonance radius at the "supermaximum" of XTE J1118+480. This rather small disk may cause the inward propagation of an eccentricity wave in this low- $q$ system.

\section{Summary}

Our findings through observations of four superoutbursts of TV Crv are summarized below:

i) We accurately determined the superhump period to be $0.065028 \pm 0.000008 \mathrm{~d}$. ii) In conjunction with the orbital period in Woudt \& Warner (2003), the superhump period yields a high superhump period excess of $0.0342 \pm 0.0021$. This implies that TV Crv has a relatively large mass ratio compared with other shortperiod SU UMa systems. Using the empirical relationship for the superhump mass ratio in Patterson (2001), the mass ratio of TV Crv is estimated to be $q=0.16 \pm 0.01$.

iii) TV Crv experiences two types of superoutbursts; one with a precursor and the other without. This behaviour can be interpreted with the refined thermal-tidal instability model if TV Crv has a relatively large mass ratio in spite of its short orbital period.

iv) We show that the superhump period derivative is variable in distinct superoutbursts. The difference is apparently related to the presence/absence of a precursor.

v) We propose that the eccentric mode keeps excited when the accretion disk remains larger than the 3:1 resonance radius. This scenario can explain the behaviour of TV Crv, and furthermore be consistent with systematically large period derivatives in superoutbursts without a precursor.

Acknowledgements. We greatly appreciate valuable comments made by Dr. Shin Mineshige. This work is supported by the Grant-in-Aid for the 21st Century COE "Center for Diversity and Universality in Physics" from the Ministry of Education, Culture, Sports, Science and Technology (MEXT) of Japan. R.M. acknowledges grant Fondecyt 1030707. P.N. acknowledges the Curry Foundation and the AAVSO. This work is partly supported by a grant-in aid from the Japanese Ministry of Education, Culture, Sports, Science and Technology (Nos. 13640239, 15037205). Part of this work is supported by a Research Fellowship of the Japan Society for the Promotion of Science for Young Scientists.

\section{References}

Augusteijn, T., van Kerkwijk, M. H., \& van Paradijs, J. 1993, A\&A, 267, L55

Baba, H., Kato, T., Nogami, D., et al. 2000, PASJ, 52, 429

Bateson, F. M. 1977, New Zealand J. Sci., 20, 73

Downes, R. A., Webbink, R. F., Shara, M. M., et al. 2001, PASP, 113, 764

Haefner, R., Schoembs, R., \& Vogt, N. 1979, A\&A, 77, 7

Harvey, D. A., \& Patterson, J. 1995, PASP, 107, 1055

Howell, S. B., DeYoung, J. A., Mattei, J. A., et al. 1996a, AJ, 111, 2367

Howell, S. B., Reyes, A. L., Ashley, R., Harrop-Allin, M. K., \& Warner, B. 1996b, MNRAS, 282, 623

Imada, A., Kato, T., Uemura, M., et al. 2004, PASJ, submitted

Ishioka, R., Kato, T., Uemura, M., et al. 2001, PASJ, 53, 905

Ishioka, R., Kato, T., Uemura, M., et al. 2003, PASJ, 55, 683

Ishioka, R., Uemura, M., Matsumoto, K., et al. 2002, A\&A, 381, L41

Kato, T. 1993, PASJ, 45, L67

Kato, T. 1995, IBVS, 4152

Kato, T. 1996, IBVS, 4369

Kato, T. 1997, PASJ, 49, 583

Kato, T. 2002, PASJ, 54, 87

Kato, T., Nogami, D., Baba, H., et al. 1996, PASJ, 48, L21

Kato, T., Nogami, D., Matsumoto, K., \& Baba, H. 1997 (ftp://vsnet. kusastro.kyoto-u.ac.jp/pub/vsnet/ preprints/EG_Cnc/)

Kato, T., Nogami, D., Masuda, S., \& Baba, H. 1998, PASP, 110, 1400 
Kato, T., Kiyota, S., Novák, R., \& Matsumoto, K. 1999, IBVS, 4794

Kato, T., Matsumoto, K., Nogami, D., Morikawa, K., \& Kiyota, S. 2001a, PASJ, 53, 893

Kato, T., Sekine, Y., \& Hirata, R. 2001b, PASJ, 53, 1191

Kato, T., Uemura, M., Ishioka, R., \& Pietz, J. 2002, PASJ, 54, 1017

Kato, T., Bolt, G., Nelson, P., et al. 2003a, MNRAS, 341, 901

Kato, T., Nelson, P., Stockdale, C., et al. 2003b, MNRAS, 347, 861

Kato, T., Santallo, S., Bolt, G., et al. 2003c, MNRAS, 339, 861

Kato, T., Nogami, D., Matsumoto, K., \& Baba, H. 2004a, PASJ, 56, S109

Kato, T., Uemura, M., Ishioka, R., et al. 2004b, PASJ, in press

Kuulkers, E. 2001, Astron. Nachr., 322, 9

Kuulkers, E., van Amerongen, S., van Paradijs, J., \& Rottgering, H. 1991, A\&A, 252, 605

Kuulkers, E., Knigge, C., Steeghs, D., Wheatley, P. J., \& Long, K. S. 2002, in The Physics of Cataclysmic Variables and Related Objects, ed. B. T. Gansicke, K. Beuermann, \& K. Reinsch (San Francisco: ASP), 443

Levy, D. H., Howell, S. B., Kreidl, T. J., Skiff, B. A., \& Tombaugh, C. W. 1990, PASP, 102, 1321

Lubow, S. H. 1992, ApJ, 401, 317

Marino, B. F., \& Walker, W. S. G. 1979, in Changing Trends in Variable Star Research, ed. F. M. Bateson, J. Smak, \& J. H. Urch (Univ. of Waikato, Hamilton, N. Z.), IAU Coll., 46, 29

Mineshige, S., Hirose, M., \& Osaki, Y. 1992, PASJ, 44, L15

Mineshige, S., Liu, B., Meyer, F., \& Meyer-Hofmeister, E. 1998, PASJ, 50, L5

Nogami, D., Kato, T., Baba, H., et al. 1997a, ApJ, 490, 840

Nogami, D., Masuda, S., \& Kato, T. 1997b, PASP, 109, 1114

Nogami, D., Baba, H., Kato, T., \& Novák, R. 1998, PASJ, 50, 297

Nogami, D., Baba, H., Matsumoto, K., \& Kato, T. 2003a, PASJ, 55, 483

Nogami, D., Uemura, M., Ishioka, R., et al. 2003b, A\&A, 404, 1067

Nogami, D., Uemura, M., Ishioka, R., et al. 2004a, PASJ, 56, S99

Nogami, D., Uemura, M., Ishioka, R., Kato, T., \& Pietz, J. 2004b, PASJ, 56, S155

Olech, A. 1997, Acta Astron., 47, 281

Olech, A., Schwarzenberg-Czerny, A., Kȩdzierski, P., et al. 2003, Acta Astron., 53, 1750

Olech, A., Cook, L. M., Zloczewski, K., et al. 2004, Acta Astron., submitted [arXiv: astro-ph/0408080]
Osaki, Y. 1985, A\&A, 144, 369

Osaki, Y. 1989, PASJ, 41, 1005

Osaki, Y., \& Meyer, F. 2003, A\&A, 401, 325

Patterson, J. 1979, AJ, 84, 804

Patterson, J. 2001, PASP, 113, 736

Patterson, J., Bond, H. E., Grauer, A. D., Shafter, A. W., \& Mattei, J. A. 1993, PASP, 105, 69

Patterson, J., Jablonski, F., Koen, C., O’Donoghue, D., \& Skillman, D. R. 1995, PASP, 107, 1183

Patterson, J., Thorstensen, J. R., Kemp, J., et al. 2003, PASP, 115, 1308

Pojmanski, G. 2002, Acta Astron., 52, 397

Schoembs, R. 1986, A\&A, 158, 233

Semeniuk, I. 1980, A\&AS, 39, 29

Semeniuk, I., Nalezyty, M., Gembara, P., \& Kwast, T. 1997, Acta Astron., 47, 299

Smak, J. 1996, in Cataclysmic Variables and Related Objects, ed. A. Evans \& J. H. Wood (Dordrecht: Kluwer Academic Publisher), IAU Coll., 158, 45

Stellingwerf, R. F. 1978, ApJ, 224, 953

Stolz, B., \& Schoembs, R. 1984, A\&A, 132, 187

Tappert, C., Mennickent, R. E., Arenas, J., Matsumoto, K., \& Hanuschik, R. W. 2003, A\&A, 408, 651

Thorstensen, J. R., Fenton, W. H., Patterson, J. O., et al. 2002, ApJ, 567, L49

Udalski, A. 1990, AJ, 100, 226

Uemura, M., Kato, T., Pavlenko, E., Baklanov, A., \& Pietz, J. 2001, PASJ, 53, 539

Uemura, M., Kato, T., Ishioka, R., et al. 2002a, PASJ, 54, 599

Uemura, M., Kato, T., Ishioka, R., et al. 2002b, PASJ, 54, L15

Uemura, M., Kato, T., Matsumoto, K., et al. 2002c, PASJ, 54, 285

Uemura, M., Kato, T., Ishioka, R., et al. 2004, PASJ, 56, S141

van Amerongen, S., Bovenschen, H., \& van Paradijs, J. 1987, MNRAS, 229, 245

Wagner, R. M., Foltz, C. B., Shahbaz, T., et al. 2001, ApJ, 556, 42

Warner, B. 1985, in Interacting Binaries, ed. P. P. Eggelton \& J. E. Pringle (Dordrecht: D. Reidel Publishing Co.), 367

Whitehurst, R. 1988, MNRAS, 232, 35

Whitehurst, R. 1994, MNRAS, 266, 35

Woudt, P. A., \& Warner, B. 2003, MNRAS, 340, 1011 